\title{
Recent incidences and differential trends of thyroid cancer in the USA
}

\author{
Yushan Mao and Mingzhao Xing \\ Laboratory for Cellular and Molecular Thyroid Research, Division of Endocrinology, Diabetes and \\ Metabolism, Johns Hopkins University School of Medicine, Maryland, MD, USA
}

Correspondence should be addressed to $M$ Xing Email mxing1@jhmi.edu

\begin{abstract}
The incidence rate of thyroid cancer has been rising rapidly in recent decades; however, its trend remains unclear. To investigate this, we analyzed the database of the Surveillance, Epidemiology and End Results (SEER) 13, 1992-2012 in the USA, particularly focusing on conventional papillary thyroid cancer (CPTC) and follicular variant of PTC (FVPTC). Of the 75,992 thyroid cancers, $61.3 \%$ were CPTC and $25.7 \%$ were FVPTC, and their incidence rates (IRs) were significantly increased from 1992 to $2012(P$ all $<0.001)$, with CPTC being 2.4 times of FVPTC $(P<0.001)$ and the overall average annual percent change (AAPC) of incidence being $6.3 \%$ in the former and $5.3 \%$ in the latter. IRs were increased in all thyroid cancers, albeit most dramatically in PTC, in virtually all ethnic/ demographic groups in recent two decades; however, the incidence trends varied among different thyroid cancers, particularly differentiable between CPTC and FVPTC. For example, Joinpoint analyses revealed that the APC of CPTC before 1996 was $1.5 \%$ $(P>0.05)$, which jumped to $6.8 \%(P<0.05)$ after 1996, whereas the APC of FVPTC before 2000 was $6.6 \%(P<0.05)$, which dropped to $4.8 \%(P<0.05)$ after 2000 . IRs and incidence trends of PTC were uneven among different ethnic/demographic groups, as exemplified by the lower IRs of both PTC variants in the Black females than in non-Hispanic White females but higher AAPCs of incidence in the former than in the latter. Interestingly, the data also suggest that the rise in the IRs of PTC is becoming plateaued in the most recent 2 years. These novel observations are helpful in understanding the incidence and incidence trends of thyroid cancer.
\end{abstract}
Key Words
- thyroid cancer
- incidence rate
- incidence trend
- conventional papillary thyroid cancer
- follicular variant of papillary thyroid cancer

\section{Introduction}

The incidence rate of thyroid cancer has been steadily rising worldwide in recent decades (Kilfoy et al. 2009, Pellegriti et al. 2013). In the USA, the overall incidence rate of thyroid cancer rose by $6.6 \%$ annually between 2000 and 2009, which was the highest among all cancers(Siegeletal.2013). TheSurveillance, Epidemiology and End Results (SEER) program has reported the age-adjusted incidence rate of thyroid cancer to be 13.5 per 100,000 people in the general population in 2015 (Howlader et al. 2015) and a 3.8-fold increase since
1973 (Davies \& Welch 2006). The number of new cases of thyroid cancer and thyroid cancer-related deaths was estimated to be 62,450 and 1950 in 2015, respectively, in the USA (Howlader et al. 2015). This increase in the incidence rate of thyroid cancer is primarily due to the rise in the incidence rate of papillary thyroid cancer (PTC), which accounts for $80-90 \%$ of all thyroid malignancies (Hundahl et al. 1998, Howlader et al. 2015). Other types of thyroid cancer include relatively uncommon follicular thyroid cancer (FTC), anaplastic

Published by Bioscientifica Ltd. 
thyroid cancer (ATC), and medullary thyroid cancer (MTC) (Xing 2013).

PTC can be histologically classified into several variants, and the most common variants are conventional PTC (CPTC) and follicular variant of PTC (FVPTC), which account for $>90 \%$ of all PTC variants (Shi et al. 2016). CPTC is a well-established classical PTC. FVPTC was initially described as alveolar variant of PTC over half a century ago (Crile \& Hazard 1953, Yu et al. 2013) and became widely accepted in 1980s (Rosai et al. 1983, Carcangiu et al. 1985). Following its inclusion in the World Health Organization classification of thyroid neoplasia in 1989 (Hedinger et al. 1989), the SEER program started including FVPTC in reporting on thyroid cancer in 1990 (Hodgson et al. 2004). Both similarities and dissimilarities in clinicopathological behavior between CPTC and FVPTC were reported, with the 10-year disease-specific patient survival rate being 97\% in the former and 98\% in the latter (Ito et al. 2008, $\mathrm{Yu}$ et al. 2013). There are also genetic similarities and dissimilarities between CPTC and FVPTC (Xing 2013).

There have been many published epidemiological studies on thyroid cancer; however, few of them specifically examined the incidence trends of thyroid cancer, including CPTC and FVPTC. Accurate and updated knowledge of the incidence trends of CPTC and FVPTC would be useful in better understanding the two most common PTC variants and in assisting public health planning and clinical practice. To this end, this study extended previous studies using the most recently updated and most comprehensive SEER 13 registry dataset to perform an in-depth analysis of the incidence rate (IR) of various thyroid cancers with a particular focus on the incidence trends of the two most common thyroid cancers (CPTC and FVPTC) in various clinicopathological and demographical settings in the USA from 1992 to 2012.

\section{Materials and methods}

\section{Data collection and inclusion}

The SEER 13 registry was used in this study. This is a retrospective cancer cohort from 13 registries, which began contributing cases in 1992, encompassing 13.4\% of the US census and covering Atlanta, Alaska Natives, Connecticut, Detroit, Hawaii, Iowa, Los Angeles, New Mexico, Rural Georgia, San Francisco-Oakland, San Jose-Monterey, Seattle-Puget Sound, and Utah (http:// seer.cancer.gov/registries/terms.html) (SEER Program
2014 submission). Both CPTC and FVPTC were specifically classified and included in this cohort. Other registries include SEER 9, which began contributing cases in 1973 but only covered nine registries and $9.4 \%$ of the US population, and SEER 18, which covered 18 registries and $27.8 \%$ of the US population but began lately in 2000. As the incidence of thyroid cancer was relatively stable until early 1990's, after which it has been rising substantially, we chose to use SEER 13 for this study.

All incidence cases with thyroid cancer were identified using the primary site code of 73.9 for thyroid in combination with the International Classification of Diseases for Oncology, 3rd Revision (ICD-O-3) (Fritz 2000). Based on the corresponding histological codes, PTC included (i) CPTC: 8050 (papillary carcinoma not otherwise specified, NOS), 8260 (papillary adenocarcinoma, NOS), 8343 (papillary carcinoma, encapsulated), and 8341 (papillary microcarcinoma); (ii) FVPTC: 8340 (papillary carcinoma, follicular variant); and (iii) other variants: 8052 (papillary squamous cell carcinoma), 8130 (papillary transitional cell carcinoma), 8342 (papillary carcinoma, oxyphilic cell), 8344 (papillary carcinoma, columnar cell), 8450 (papillary cystadenoma, NOS), 8451 (papillary cystadenoma, borderline malignancy), and 8452 (papillary cystic tumor). FTC included 8290 (oxyphilic adenocarcinoma), 8330 (follicular adenocarcinoma, NOS), 8331 (follicular adenocarcinoma well differentiated), 8332 (follicular adenocarcinoma trabecular), and 8335 (follicular carcinoma, minimal invasive). MTC included 8345 (medullary carcinoma with amyloid stroma), 8346 (mixed medullary-follicular carcinoma), 8347 (mixed medullary-papillary carcinoma), and 8510 (medullary carcinoma, NOS). ATC included 8021 (carcinoma, anaplastic type, NOS).

Incidence trends were analyzed by various histopathological and demographic parameters, including tumor size, patient sex, ethnicity/race, and SEER historic stage A. SEER ethnic/racial groups included Hispanic Whites, non-Hispanic Whites, Blacks, and Asian/others (including Asians/Pacific Islanders and American Indians/Alaskan Natives). SEER historic stage A was a classification system used to define the invasion/metastatic status of thyroid cancer, including localized (limited to the thyroid gland), regional (limited to surrounding tissues in the neck), and distant (or systemic) diseases. Tumor size was referred to the greatest diameter of the tumor recorded on surgical pathology reports, which was categorized as $\leq 1$; $>1$ and $\leq 2$; $>2$ and $\leq 4$; and $>4 \mathrm{~cm}$ based on the Extent of

Published by Bioscientifica Ltd 
Disease-10 codes for 1992-2003 and the Collaborative Staging codes for 2004-2012.

\section{Data analysis}

IR was calculated using SEER*Stat 8.2.1 (www.seer.cancer. gov/seerstat) and expressed as per 100,000 populationyear. Age-adjusted IRs were standardized to the 2000 US population. Incidence rate ratio (IRR) was used to compare IRs, where a given rate of a group was compared with a referent group rate (Tiwari et al. 2006). IRs were reported if there were $\geq 10$ cases in a category and IRRs were reported if both rates were $\geq 10$ cases.

Joinpoint analysis, which was patient age adjusted, was performed to investigate the incidence trends using Joinpoint Regression Program 4.0.4 from the Surveillance Research Program of the National Cancer Institute. This helped to identify inflection points (so-called joinpoints) where a significant change in linear slope of the trend occurred, allowing two segments to fit age-adjusted trends between 1992 and 2012. Each line segment was characterized with the estimated annual percentage change (APC), reflecting a change in trend within the time horizon of the line segment at a constant percentage of the rate of the previous year (Kim et al. 2000). To quantify the trend over the whole period, we computed the average annual percent change (AAPC) as a geometric weighted average of the APCs from the Joinpoint model. This represented a summary measure of the trend over the study period. If an AAPC lied entirely within a single joinpoint segment, the AAPC was equal to the APC for that segment. An associated $P<0.05$ represented the likelihood that the APC or AAPC was significantly different from zero. Pairwise comparison parallel test by Joinpoint software was used to compare the trends among groups. Parallelism test was used to examine whether regression mean functions (slope of the change in trend) were similar in direction. A $P$ value $<0.05$ on this test suggested that the two trends in terms of AAPCs compared were significantly different from each other. Other analyses were performed using STATA software, version 12.0 (STATA, College Station, TX, USA). All $P$ values were two-sided and a value $<0.05$ was treated as significant.

\section{Results}

\section{Proportion and incidence trends of thyroid cancers}

Among the 77,848 cases of thyroid cancer recorded in SEER $13,97.6 \%(75,992)$ had microscopically confirmed histology, which were included for the analyses in this study. Figure 1A shows the proportions of various
A

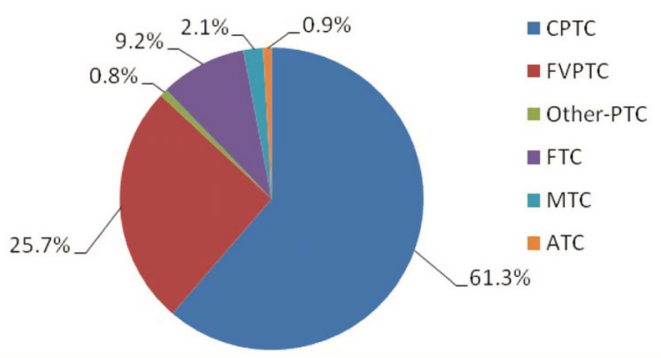

B

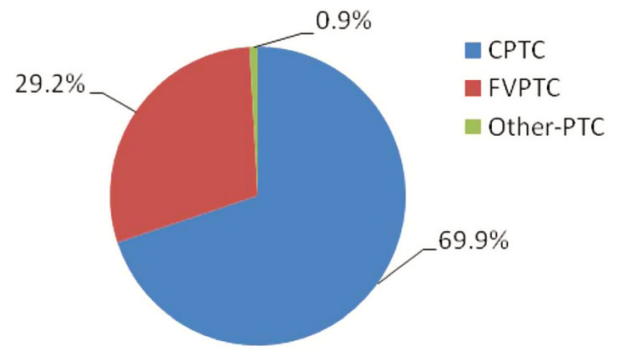

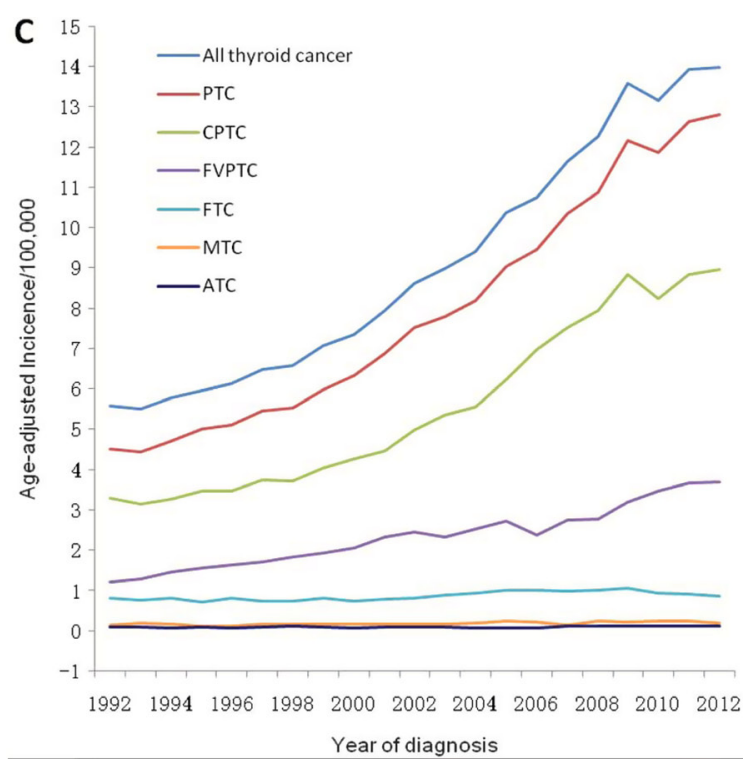

\section{Figure 1}

Proportions and incidence rate trends of various thyroid cancers in the population of USA from 1992 to 2012. (A) Percentage proportions of various histological types of thyroid cancer. (B) Percentage proportions of various variants of papillary thyroid cancer. (C) Thyroid cancer incidence trends. PTC, papillary thyroid cancer; CPTC, conventional PTC; FVPTC, follicular variant of PTC; FTC, follicular thyroid cancer; MTC, medullary thyroid cancer; ATC, anaplastic thyroid cancer. 
Table 1 Age-adjusted incidence rates (per 100,000) of thyroid cancer and their average annual percentage change between 1992 and 2012 in the USA.

\begin{tabular}{|c|c|c|c|c|c|c|c|}
\hline \multirow[b]{2}{*}{ Histological type } & \multirow[b]{2}{*}{$n$} & \multicolumn{3}{|c|}{ Age-adjusted incidence rate } & \multirow[b]{2}{*}{$P$ for trend } & \multirow[b]{2}{*}{ AAPC } & \multirow[b]{2}{*}{$95 \% \mathrm{Cl}$} \\
\hline & & Overall & $\begin{array}{l}\text { Year } \\
1992\end{array}$ & $\begin{array}{l}\text { Year } \\
2012\end{array}$ & & & \\
\hline All thyroid cancer & 75,992 & 9.31 & 5.57 & 13.98 & $<0.001$ & $5.5^{*}$ & $5.2-5.8$ \\
\hline PTC & 66,695 & 8.15 & 4.51 & 12.82 & $<0.001$ & $6.1 *$ & $5.8-6.4$ \\
\hline CPTC & 46,603 & 5.69 & 3.30 & 8.96 & $<0.001$ & $6.3^{*}$ & $5.8-6.9$ \\
\hline FVPTC & 19,520 & 2.39 & 1.21 & 3.70 & $<0.001$ & $5.3^{*}$ & $4.8-5.8$ \\
\hline FTC & 7,013 & 0.87 & 0.80 & 0.86 & $<0.001$ & $1.5^{*}$ & $0.8-2.2$ \\
\hline MTC & 1,579 & 0.20 & 0.16 & 0.19 & $<0.001$ & $2.3^{*}$ & $1.2-3.4$ \\
\hline ATC & 705 & 0.09 & 0.09 & 0.11 & 0.027 & $1.6^{*}$ & $0.3-2.8$ \\
\hline
\end{tabular}

Notes: PTC, papillary thyroid cancer; CPTC, conventional papillary thyroid cancer; FVPTC, follicular variant of papillary thyroid cancer; FTC, follicular thyroid cancer; MTC, medullary thyroid cancer; ATC, anaplastic thyroid cancer; $95 \% \mathrm{Cl}, 95 \%$ confidence interval; AAPC, average annual percentage change.

*Statistical significance $(P<0.05)$ was found in incidence trends as indicated.

histological types of thyroid cancer, with PTC being the most common, accounting for $87.8 \%$ of all thyroid cancers, and FTC the second common, accounting for 9.2\% of all thyroid cancers. CPTC and FVPTC accounted for $61.3 \%$ and $25.7 \%$ of all thyroid cancers respectively. CPTC and FVPTC accounted for $69.9 \%$ and $29.2 \%$ of all PTC variants respectively (Fig. 1B). Fig. 1C shows the trends in IRs of all histological types of thyroid cancer, including CPTC and FVPTC. There was a steady rise in the IR most prominently with total PTC, CPTC, and FVPTC between 1992 and 2012; however, the rising was steeper with CPTC than FVPTC.

Table 1 summarizes age-adjusted IRs and AAPC of thyroid cancer from 1992 to 2012. The results included the overall trend across the 20 years and the difference between 1992 and 2012 at the two points in time. The incidence of overall thyroid cancer increased more than 2.5-fold during this period $(P<0.001$ for trend) with the AAPC being 5.5\% (95\% CI, 5.2-5.8). This progressive increase was nearly entirely attributable to an increase in PTC, which was increased by 2.8 fold $(P<0.001$ for trend) with an AAPC of $6.1 \%(95 \%$ CI, 5.8-6.4). The incidence of CPTC was increased by 2.7-fold from 1992 to 2012 ( $P<0.001$ for trend) with an AAPC of $6.3 \%$ (95\% CI, 5.8-6.9). The incidence of FVPTC was increased by over three-fold $(P<0.001$ for trend) with the AAPC being 5.3\% (95\% CI, 4.8-5.8) during the same period. There was also a significant increase in the incidence of FTC, MTC (both $P<0.001$ for trend), and ATC $(P=0.027$ for trend), although the overall trends for these three thyroid cancers were relatively slow, with AAPC being 1.5\% (95\% CI, $0.8-2.2$ ), $2.3 \%$ (95\% CI, 1.2-3.4), and $1.6 \%$ (95\% CI, 0.3-2.8) respectively. Thus, the incidence change occurred mainly with CPTC and FVPTC, which were the focus of the further analyses.

Table 2 Joinpoint analysis of the age-adjusted incidence and trend of CPTC and FVTC by patient sex between 1992 and 2012 in the USA.

\begin{tabular}{|c|c|c|c|c|c|c|c|c|}
\hline $\begin{array}{l}\text { Sex and } \\
\text { PTC type }\end{array}$ & $n$ & $\begin{array}{c}\text { IR } \\
(95 \% \mathrm{Cl})\end{array}$ & $\begin{array}{c}\text { IRR } \\
(95 \% \mathrm{Cl})\end{array}$ & $\begin{array}{c}\text { APC1 } \\
(95 \% \mathrm{Cl})\end{array}$ & Joinpoint & $\begin{array}{c}\text { APC2 } \\
(95 \% \mathrm{Cl})\end{array}$ & $\begin{array}{c}\text { AAPC } \\
(95 \% \mathrm{Cl})\end{array}$ & Parallelisma \\
\hline \multicolumn{9}{|l|}{ Both sex } \\
\hline FVPTC & 19,520 & $2.39(2.36-2.43)$ & 1.00 & $6.6(4.0-9.4)^{\mathrm{b}}$ & 2000 & $4.8(3.8-5.8)^{\mathrm{b}}$ & $5.3(4.8-5.8)^{\mathrm{b}}$ & 0.006 \\
\hline СРTC & 46,603 & $5.69(5.64-5.74)$ & $2.38(2.34-2.42)^{c}$ & $1.5(-6.1-9.7)$ & 1996 & $6.8(6.1-7.5)^{b}$ & $6.3(5.8-6.9)^{b}$ & \\
\hline \multicolumn{9}{|l|}{ Female } \\
\hline FVPTC & 15,338 & $3.66(3.60-3.72)$ & 1.00 & $5.4(4.9-6.0)^{b}$ & - & & $5.4(4.9-6.0)^{b}$ & 0.005 \\
\hline СРТC & 35,610 & $8.47(8.38-8.56)$ & $2.32(2.27-2.36)^{c}$ & $6.5(5.9-7.1)^{b}$ & - & & $6.5(5.9-7.1)^{b}$ & \\
\hline \multicolumn{9}{|l|}{ Male } \\
\hline FVPTC & 4,182 & $1.09(1.05-1.12)$ & 1.00 & $4.3(3.2-5.4)^{\mathrm{b}}$ & 2008 & $7.9(1.5-14.7)^{\mathrm{b}}$ & $4.9(4.2-5.6)^{\mathrm{b}}$ & 0.013 \\
\hline CPTC & 10,993 & $2.84(2.78-2.89)$ & $2.61(2.52-2.71)^{c}$ & $-4.0(-16.3-10.2)$ & 1995 & $6.3(5.6-7.1)^{\mathrm{b}}$ & $5.7(5.0-6.4)^{b}$ & \\
\hline
\end{tabular}

Notes: $95 \%$ Cl, 95\% confidence interval; APC, annual percentage change; APC1, the APC from 1992 to the year of joinpoint; APC2, the APC from the year of joinpoint to 2012; AAPC, average annual percentage change; CPTC, conventional papillary thyroid cancer; FVPTC, follicular variant of papillary thyroid cancer; IR, incidence rate; IRR, incidence rate ratio.

a $P$ value $<0.05$ on the parallelism test, indicating that the trends (slopes) between CPTC and FVPTC were significantly different.

bAPC or AAPC was significantly different from 0 (two-sided, $P<0.05$ ).

$c P$ value $<0.05$.

http://erc.endocrinology-journals.org

DOI: 10.1530/ERC-15-0445
() 2016 Society for Endocrinology Printed in Great Britain
Published by Bioscientifica Ltd. 

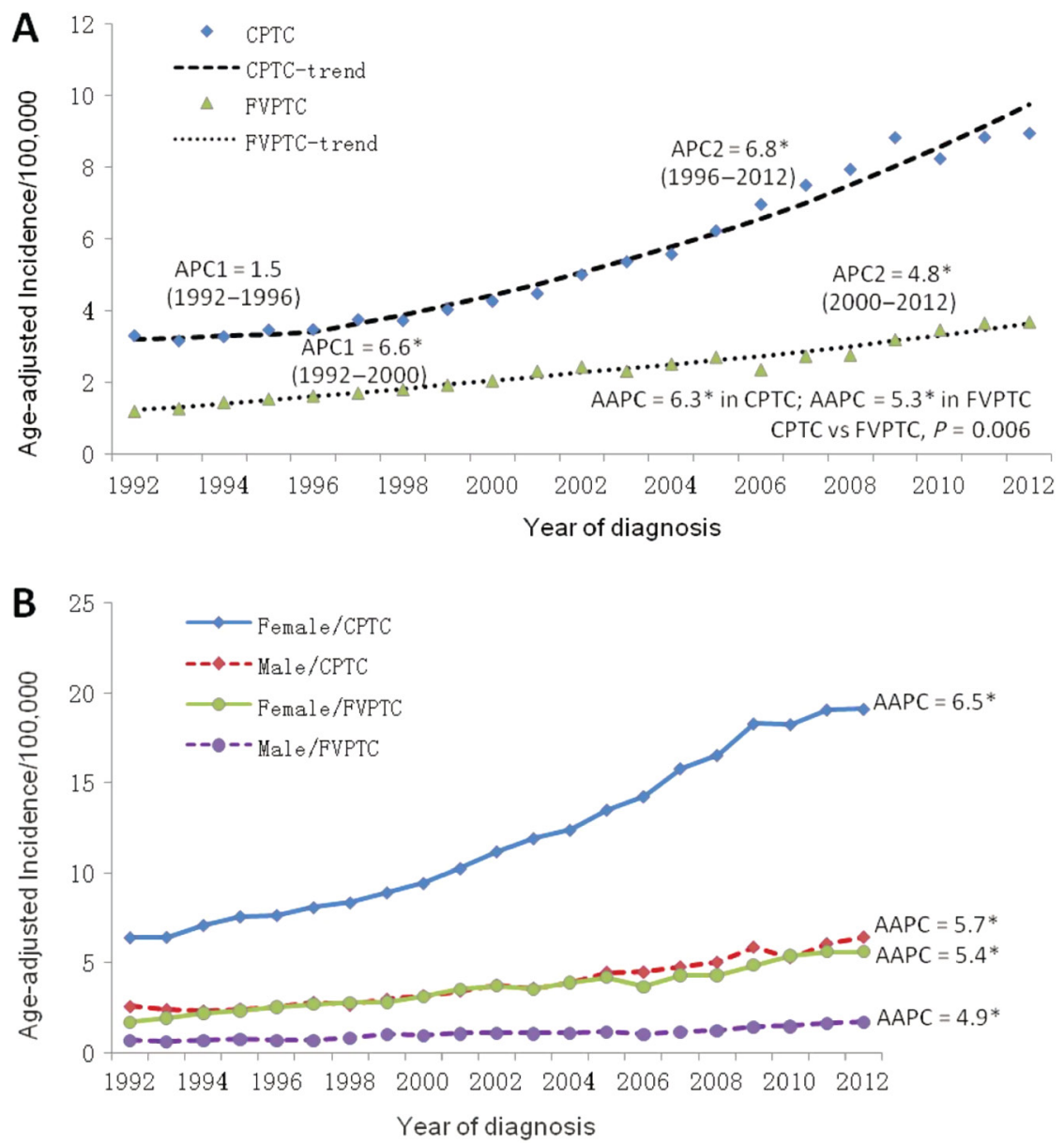
Figure 2
Trends of age-adjusted incidences of CPTC and FVPTC. (A) Analysis using Joinpoint regression and comparison analysis. (B) Analysis by sex. *The AAPC was significantly different from 0 (two-sided, $P<0.05$ ). CPTC, conventional papillary thyroid cancer; FVPTC, follicular variant of papillary thyroid cancer; APC, annual percentage change; AAPC, average annual percentage change.

\section{Incidence trends of CPTC and FVPTC and their patterns by patient sex}

The patient demographic characteristics of the CPTC and FVPTC groups are presented in Supplementary Table 1 (see section on supplementary data given at the end of this article). During the period of 1992-2012, a total of 46,603 cases of CPTC (IR=5.69, 95\% CI, 5.64-5.74) and 19,520 cases of FVPTC (IR=2.39, 95 CI, 2.36-2.43) were reported; the IRR of CPTC/FVPTC was 2.38 (95\% CI, $2.34-2.42, P<0.05)$, which was similar to the IRRs when male and female patients were examined separately (2.61 and 2.32 respectively) (Table 2 ).

The over rising incidence curves between 1992 and 2012 were steeper in CPTC than FVPTC in patients of both sexes or either sex alone (Fig. 2). The incidence trends from Joinpoint regression analyses demonstrated that age-adjusted incidence rates of both CPTC and FVPTC continuously increased between 1992 and 2012. However, one joinpoint emerged in incidence trend line of CPTC or FVPTC between 1992 and 2012 on the analysis of patients of both sexes (Fig. 2A). On the overall analysis of all cases (Table 2), the APC of CPTC between 1992 and 1996 was $1.5 \%(P>0.05)$, which jumped to $6.8 \%(P<0.05)$ between 1996 and 2012 , whereas the APC of FVPTC between 1992 and 2000 was $6.6 \%$ $(P<0.05)$, which dropped to $4.8 \%(P<0.05)$ between 2000 and 2012. A parallelism test indicated that the incidence trends of CPTC and FVPTC on the Joinpoint regression were significantly different $(P=0.006)$.

Fig. 2B and Table 2 show the incidence trends by patient sex for CPTC and FVPTC. When the two sexes were separated, joinpoints emerged in male sex with CPTC and FVPTC (Table 2). Specifically, the APC of CPTC in males between 1992 and 1995 was $-4.0 \%(P>0.05)$, which robustly jumped to $6.3 \%(P<0.05)$ between 1995 and 2012. The APC of FVPTC in males was $4.3 \%(P<0.05)$ before 2008, which was moderately increased to $7.9 \%$ $(P<0.05)$ after 2008 . There was no clear joinpoint in females with CPTC or FVPTC, indicating a steady increase in the incidence of both cancers over this period in this population (AAPC $=6.5 \%$ and $5.4 \%$, respectively, $P<0.05$ ). However, a parallelism test showed a significant difference in the incidence trend between CPTC and FVPTC, higher

Published by Bioscientifica Ltd 


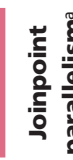

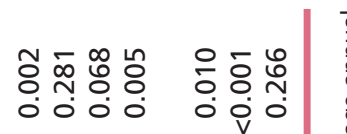
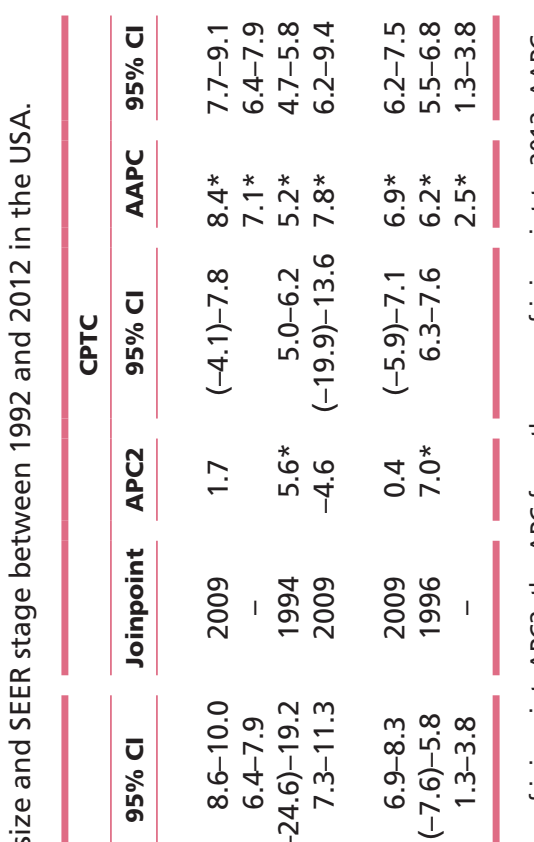

in the former than in the latter, both in males $(P=0.013)$ and in females $(P=0.005)$.

\section{Incidence trends of CPTC and FVPTC by ethnicity/race and sex}

We next analyzed the incidence patterns of CPTC and FVPTC by ethnicity/race and sex. As shown in Supplementary Table 2, the IRRs of females/males were 3.22-4.25 for FVPTC and 2.83-3.53 for CPTC; all were significant for each ethnic/racial group. The IRs of FVPTC in females were significantly higher in non-Hispanic Whites, Hispanic Whites, and Asian/others than Black females with IRRs of non-Black/Black being 1.11-1.48, which were all significant $(P<0.005)$. The IRRs of non-Black/Black for CPTC in both females and males were 2.12-2.76, which were all significant $(P<0.001)$. Although all the IRRs of nonBlack/Black for FVPTC in males were $>1.0$, it was significant only in non-Hispanic Whites (IRR=1.61, $P<0.001$ ).

We also analyzed the race-specific time trend of ageadjusted incidence of CPTC and FVPTC by sex from 1992 to 2012. During the 20-year period, the incidence of CPTC or FVPTC significantly increased in every ethnicity/race group and both sexes. Specifically, as shown in Supplementary Table 3 and Supplementary Fig. 1 (see section on supplementary data given at the end of this article), in females, the AAPC of CPTC incidence was 7.9\% (95\% CI 6.7-9.1, $P<0.05$ ) in Blacks, 7.0\% (95\% CI 6.4-7.6, $P<0.05)$ in non-Hispanic Whites, $5.6 \%(95 \% \mathrm{CI}$ 4.8-6.3, $P<0.05)$ in Hispanic Whites, and $4.6 \%$ (95\% CI 3.9-5.3, $P<0.05$ ) in Asian/others. The AAPC of CPTC in males was $6.5 \%(95 \%$ CI $5.7-7.1, P<0.05)$ in non-Hispanic Whites, $4.9 \%$ (95\% CI 3.5-6.3, $P<0.05$ ) in Hispanic Whites, $4.1 \%(95 \%$ CI $2.9-5.4, P<0.05)$ in Asian/others, and $2.5 \%$ (95\% CI $0.4-4.7, P<0.05)$ in Blacks. The IRs of FVPTC in both sexes were the lowest in the Blacks among virtually all the ethnic settings, whereas in both sexes, Blacks showed higher AAPCs of FVPTC (female $6.7 \%$ and male $8.2 \%$ ) than non-Hispanic Whites (female $6.1 \%$ and male $5.3 \%$ ), Hispanic Whites (female $4.4 \%$ and male $4.1 \%$ ), and Asian/others (female $2.8 \%$ and male $2.4 \%$ ). Thus, for both CPTC and FVPTC, while Black females had a lower IR than non-Hispanic White females, the former had a higher AAPC than the latter.

The presence of joinpoints varied in different settings. As shown in Supplementary Table 3 and Supplementary Fig. 1, for FVPTC, there was no joinpoint in all the ethnicity/race groups in both females and males except for the non-Hispanic White females. APC of FVPTC before the joinpoint in 1996 was the highest

Published by Bioscientifica Ltd. 


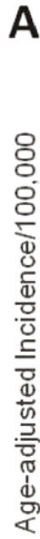
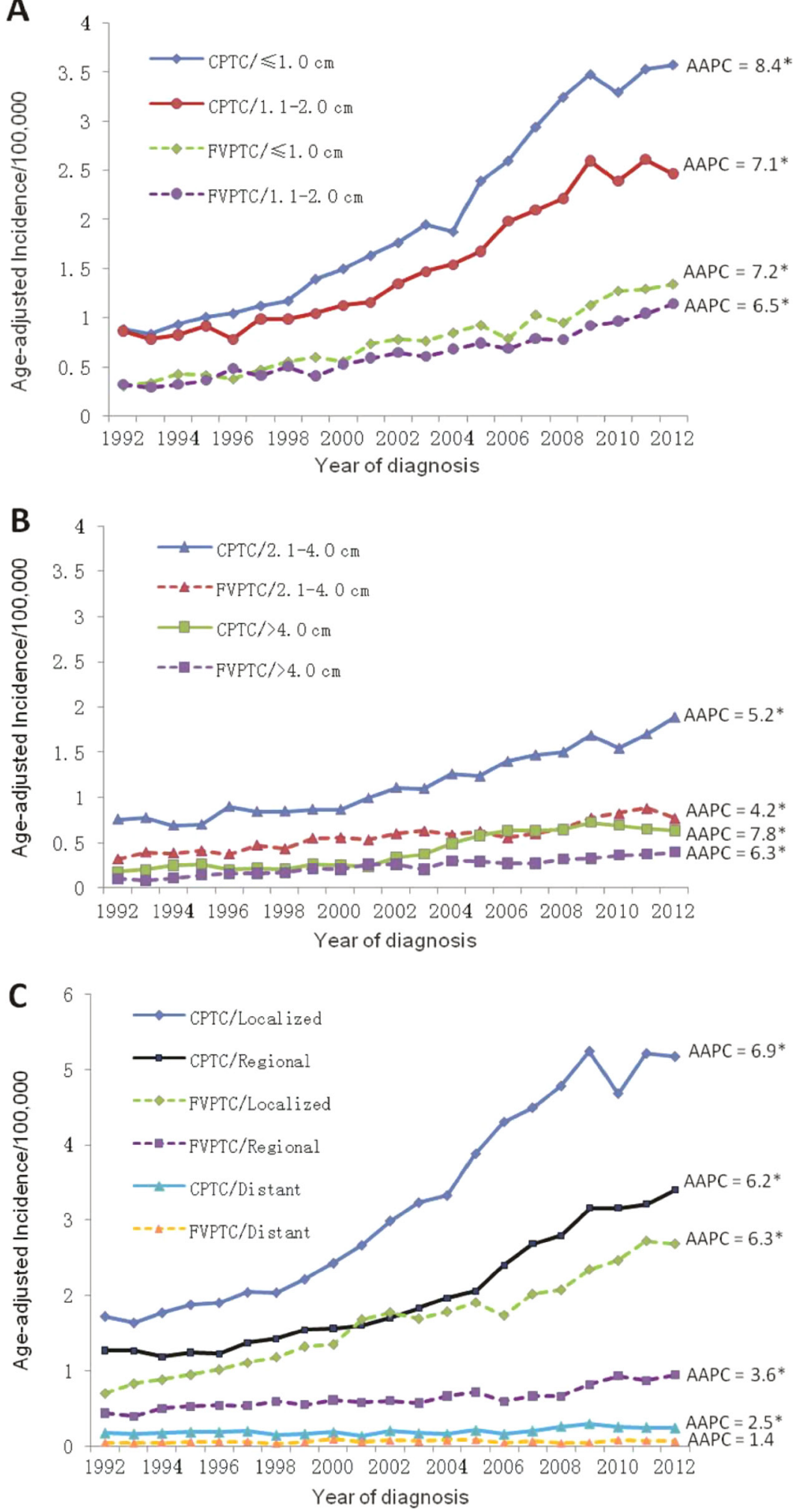

Figure 3

Trends of age-adjusted incidence of CPTC and FVPTC by tumor size and SEER tumor stage A. (A) By tumor size $<1.0 \mathrm{~cm}$ and $1.1-2.0 \mathrm{~cm}$. (B) By tumor size $2.1-4.0 \mathrm{~cm}$ and $>4.0 \mathrm{~cm}$. (C) By SEER tumor stage A, 1992-2012. *The AAPC was significantly different from 0 (two-sided, $P<0.05$ ). CPTC, conventional papillary thyroid cancer; FVPTC, follicular variant of papillary thyroid cancer; AAPC, average annual percentage change.

in non-Hispanic White females (12.5\%) among all groups, which dropped to $5.6 \%$ after 1996. For CPTC, in Black females, APC was $-1.2 \%$ before 1997, which rose to $9.2 \%$ after 1997; in non-Hispanic White females, the joinpoint occurred in the most recent year of 2009, with APC being $7.7 \%$ before 2009 and $0.8 \%$ after 2009. This slowed rise in the incidence of CPTC in non-Hispanic White females is consistent with the flattened incidence 
curve in this ethnic population in the most recent 2 years (Supplementary Fig. 1). However, the overall incidence rising curves over the 20 years were steeper with CPTC than FVPTC in virtually all the ethnic backgrounds with both patient sexes (Supplementary Fig. 1).

We also performed Joinpoint parallelism test to examine the difference in time trends among different groups, as summarized in Supplementary Table 4. In females, the incidence trends of FVPTC among different ethnicity/race groups were all significantly different except for the comparison between non-Hispanic White females and Black females $(P=0.167)$. The incidence trends of CPTC were also significantly different among all ethnicity/race groups except for the comparison between Hispanic White females and Asian/other females $(P=0.133)$. As an example, the incidence trends between Black females and Hispanic White females were significantly different for both FVPTC and CPTC $(P<0.001$ for all). In males, a significant difference in incidence trends of FVPTC was also observed among various ethnic/ race groups (Supplementary Table 4).

\section{Incidence trends of CPTC and FVPTC by tumor size and SEER stage}

We also examined tumor size-specific and stage-specific incidences of CPTC and FVPTC as shown in Table 3 and Fig. 3. The changes of incidence trend were larger in small tumors than in large tumors (Fig. 3A and B) and in localized disease stage than in regional and distant disease stages (Fig. 3C), although, as shown in Table 3, the AAPCs of CPTC were significant among all tumor size groups and all SEER stage groups $(P<0.05$ for all). A significant joinpoint was identified in most of the tumor size and disease stage groups of CPTC. For example, the APC of CPTC with tumor size $2.1-4.0 \mathrm{~cm}$ was $-5.2 \%$ before 1994 , which jumped to 5.6\% after 1994, and the APC of CPTC with regional metastasis was $-1.1 \%$ before 1996 , which jumped to $7.0 \%$ after 1996 . For CPTC $>4.0 \mathrm{~cm}$, the joinpoint occurred in the most recent year of 2009, with APC being $9.3 \%$ before 2009 and $-4.6 \%$ after 2009 , representing a sharply decreased (actually negative) incidence trend in the most recent 2-3 years, consisting with the slightly downward incidence trend curve of CPTC $>4.0 \mathrm{~cm}$ from 2009 to 2012 (Fig. 3B). No joinpoint was identified in the distant metastasis group of CPTC, indicating a steadily and slow rising incidence trend from 1992 to 2012. No joinpoint was identified for FVPTC in most of the tumor size groups except for the group of tumor $>4.0 \mathrm{~cm}$; in the latter, the APC was $12.8 \%$ before 1999, which dropped to $4.6 \%$ after 1999 , indicating a slowing of the incidence trend of FVPTC $>4.0 \mathrm{~cm}$ in the most recent decade. Nevertheless, over the course of 20 years, the overall rise of the incidence occurred virtually in all the tumor size and SEER stage groups in both CPTC and FVPTC, and the rise was sharper in the former than the latter, particularly during the time before the most recent 2-3 years (Fig. 3).

\section{Discussion}

This is an analysis of the incidence and incidence trends of various thyroid cancers, particularly CPTC and FVPTC, in various ethnic and demographic settings on SEER 13, the most comprehensive recent thyroid cancer dataset in the USA, spanning 20 years from 1992 to 2012. The results showed a significant increase in age-adjusted IR of all thyroid cancers, particularly CPTC and FVPTC, over this period. The overall rising trend of CPTC incidence was most prominent and significantly higher than that of FVPTC. Although the overall incidence of FVPTC was also steadily increasing among most populations from 1992 to 2012, a slowed trend was generally seen in the recent decade compared with the earlier decade, whereas the incidence trend of CPTC was generally increased in the recent decade on the overall analysis except for certain ethnic/demographic settings in the most recent $2-3$ years. Thus, this study for the first time unveils an interesting differentiation of the incidence rise trend in the last two decades between CPTC and FVPTC - generally increased in the former and generally decreased in the latter.

This study also found a significant increase in incidence trend in all histological types of thyroid cancers, albeit most robustly in PTC. This was different than the previous results on the analysis of SEER 9 that showed no significant changes between 1973 and 2002 in the incidence of FTC, MTC, and ATC (Davies \& Welch 2006). However, this study showed that the increased rate of the incidence of FTC and MTC was much slower than that of PTC, which is consistent with other previous studies (Enewold et al. 2009, Aschebrook-Kilfoy et al. 2011). Inconsistent with other reports (Davies \& Welch 2006, Davies \& Welch 2014), this study found that the incidence trend of ATC had a small but significant increase in the recent two decades.

It was proposed that the increased incidence of PTC was attributed to the enhanced diagnostic detection in recent years (Davies \& Welch 2006, Davies \& Welch 2014). As in CPTC patients with small tumors and localized disease, in which our Joinpoint analyses revealed high

Published by Bioscientifica Ltd. 
AAPCs in the recent decade, we similarly showed that CPTC with larger tumor $(>2.0 \mathrm{~cm})$ or metastasis (local or distant) also displayed remarkably increased AAPCs in the recent decade. These results seem to be inconsistent with the detection theory but suggest that enhanced diagnostic detection may not be the only cause for the increased incidence of CPTC. If enhanced diagnostic detection was the sole cause for the increased incidence, there would likely be a decrease in the incidence trend of large or advanced thyroid cancer in the recent decade, as through enhanced diagnostic detection many tumors would have been detected at the early stage, leaving today less chance to find advanced thyroid cancer. Only APCs of the extremely large tumors (tumors $>4.0 \mathrm{~cm}$ ) of both CPTC and FVPTC were decreased, particularly in the most recent 2-3 years for CPTC, which is likely attributable to the increased diagnostic surveillance and treatment seen in the recent decades. Our finding that, for both CPTC and FVPTC, Black females had a higher AAPC than non-Hispanic White females in the recent decades is also inconsistent with the detection theory, as there is no evidence that the former ethnic group receives more aggressive diagnostic detection for thyroid cancer than the latter in the recent decade in USA. The finding of a much lower trend in the incidence rise in FTC than PTC is similarly inconsistent with the detection theory, as detection would be expected to similarly increase the clinical discovery and hence AAPC of both FTC and PTC. The differential AAPC between CPTC and FVPTC found in this study is also inconsistent with the detection theory in that increased diagnostic detection would proportionately promote the diagnosis of both CPTC and FVPTC and yield equal AAPCs for the two PTC variants. Our results are, instead, consistent with the results on SEER 9 dataset, suggesting that enhanced clinical detection could not fully explain the increase in the incidence of PTC (Li et al. 2013). Thus, these results provide further evidence that the currently proposed enhanced diagnostic detection theory cannot fully explain the rapidly rising incidence of PTC in the recent decades; it seems that an additional unknown etiology that has been preferentially driving the development of CPTC over FVPTC exists.

Interestingly, in opposite to the overall rapidly rising trend of the incidence of CPTC in non-Hispanic White females in the last two decades, the APC of CPTC in this population in the most recent $2-3$ years after 2009 stopped rising and in fact became negative, suggesting that the diagnosis of CPTC seems to start reaching a plateau in the recent 2-3 years. As the incidence of CPTC in nonHispanic White females constitute the bulk of the overall incidence of PTC in the USA, it is expectable, based on this finding, that the rapid incidence rise of PTC will halt and stay plateaued for the current and coming few years. To be consistent with this expectation is, in fact, the fact that the incidence of thyroid cancer in the USA in 2015 was very similar to that in 2014 (Howlader et al. 2015).

Our study has several strengths worth emphasizing. Previous studies mostly examined the cumulative incidences of thyroid cancers. Our study examined not only the incidences but also the incidence trends and compared them between the major PTC variants FVPTC and CPTC in various ethnic/demographic settings over the recent two decades during which enhanced diagnostic detection using ultrasonography and fineneedle aspiration biopsy was widely seen. Importantly, we used the Joinpoint regression analysis to specifically and accurately examine the incidence trends of PTC. This made it possible to identify a specific calendar year at which a significant change in the incidence trend occurred and to define the APCs/AAPCs before and after that time point. As a result, we were able to discover the remarkable differential incidence trends of CPTC and FVPTC. Thus, this study represents an improved approach of evaluating the contribution of diagnostic detection to the increased incidence of thyroid cancer in the recent decades.

Like all the previously published studies using various SEER databases, this study shares the classical limitations of investigations using such a large population-based dataset. For example, although SEER conducts regular audits to evaluate both the quality and completeness of data, some misclassification of patient information, such as that on tumor size and subtype diagnosis, in this large national database is virtually inevitable. Also, despite being the most representative thyroid cancer dataset in the USA, the SEER 13 regions represent only 13.4\% of the US population. Nevertheless, SEER 13 is a large and comprehensive recent US cohort of thyroid cancer patients, which helps minimize the above limitations.

\section{Conclusions}

This study demonstrates significantly increased IRs in all thyroid cancers, particularly CPTC and FVPTC, in virtually all ethnic/demographic groups from 1992 to 2012 in the USA; however, the incidence trends were different among different thyroid cancers, particularly sharply differentiable between CPTC and FVPTC. There were uneven IRs and incidence trends of PTC in different ethnic/demographic groups, as exemplified by

Published by Bioscientifica Ltd 
the fact that IRs of both CPTC and FVPTC were lower in the Black females than non-Hispanic White females; however, the AAPCs were higher in the former than in the latter. There is also an interesting suggestion from the most recent $2-3$ years that the incidence rise of PTC is likely becoming plateaued in the current and coming few years in the USA.

\section{Supplementary data}

This is linked to the online version of the paper at http://dx.doi.org/10.1530/ ERC-15-0445.

\section{Declaration of interest}

The authors declare that there is no conflict of interest that could be perceived as prejudicing the impartiality of the research reported.

\section{Funding}

This project was supported by the USA National Institutes of Health grants R01CA113507 and R01CA189224 to M Xing.

\section{References}

Aschebrook-Kilfoy B, Ward MH, Sabra MM \& Devesa SS 2011 Thyroid cancer incidence patterns in the United States by histologic type, 1992-2006. Thyroid 21 125-134. (doi:10.1089/thy.2010.0021)

Carcangiu ML, Zampi G, Pupi A, Castagnoli A \& Rosai J 1985 Papillary carcinoma of the thyroid. Cancer 55 805-828. (doi:10.1002/10970142(19850215)55:4<805::AID-CNCR2820550419>3.0.CO;2-Z)

Crile G Jr \& Hazard JB 1953 Relationship of the age of the patient to the natural history and prognosis of carcinoma of the thyroid. Annals of Surgery 138 33-38. (doi:10.1097/00000658-195307000-00005)

Davies L \& Welch GH 2006 Increasing incidence of thyroid cancer in the United States, 1973-2002. JAMA 295 2164-2167. (doi:10.1001/ jama.295.18.2164)

Davies L \& Welch HG 2014 Current thyroid cancer trends in the United States. JAMA: Otolaryngology Head \& Neck Surgery 140 317-322. (doi:10.1001/jamaoto.2014.1)

Enewold L, Zhu K, Ron E, Marrogi AJ, Stojadinovic A, Peoples GE \& Devesa SS 2009 Rising thyroid cancer incidence in the United States by demographic and tumor characteristics, 1980-2005. Cancer Epidemiology, Biomarkers \& Prevention 18 784-791. (doi:10.1158/1055-9965.EPI-08-0960)

Fritz AG 2000 International Classification of Diseases for Oncology, 3rd ed. Geneva, Switzerland: World Health Organization.

Hedinger C, Williams ED \& Sobin LH 1989 The WHO histological classification of thyroid tumors: a commentary on the second edition. Cancer 63 908-911. (doi:10.1002/10970142(19890301)63:5<908::aid-cncr2820630520>3.0.co;2-i)

Hodgson NC, Button J \& Solorzano CC 2004 Thyroid cancer: is the incidence still increasing? Annals of Surgical Oncology 11 1093-1097. (doi:10.1245/ASO.2004.03.066)
Howlader N, Noone AM, Krapcho M, Garshell J, Miller D, Altekruse SF, Kosary CL, Yu M, Ruhl J, Tatalovich Z, et al. 2015 SEER Cancer Statistics Review, 1975-2012, National Cancer Institute. Bethesda, MD. (available at: http://seer.cancer.gov/csr/1975-2012/, based on November 2014 SEER data submission, posted to the SEER web site). Accessed on June 10, 2015.

Hundahl SA, Fleming ID, Fremgen AM \& Menck HR 1998 A National Cancer Data Base report on 53,856 cases of thyroid carcinoma treated in the U.S., 1985-1995. Cancer 83 2638-2648. (doi:10.1002/ (SICI)1097-0142(19981215)83:12<2638::AID-CNCR31>3.0.CO;2-1)

Ito Y, Hirokawa M, Uruno T, Kihara M, Higashiyama T, Takamura Y, Miya A, Kobayashi K, Matsuzuka F \& Miyauchi A 2008 Prevalence and biological behaviour of variants of papillary thyroid carcinoma: experience at a single institute. Pathology 40 617-622. (doi:10.1080/00313020802320630)

Kilfoy BA, Zheng T, Holford TR, Han X, Ward MH, Sjodin A, Zhang Y, Bai Y, Zhu C, Guo GL, et al. 2009 International patterns and trends in thyroid cancer incidence, 1973-2002. Cancer Causes Control 20 525-531. (doi:10.1007/s10552-008-9260-4)

Kim HJ, Fay MP, Feuer EJ \& Midthune DN 2000 Permutation tests for joinpoint regression with applications to cancer rates. Statistics in Medicine 19 335-351. (doi:10.1002/(sici)10970258(20000215)19:3<335::aid-sim336>3.0.co;2-z)

Li N, Du XL, Reitzel LR, Xu L \& Sturgis EM 2013 Impact of enhanced detection on the increase in thyroid cancer incidence in the United States: review of incidence trends by socioeconomic status within the Surveillance, Epidemiology, and End Results Registry, 1980-2008. Thyroid 23 103-110. (doi:10.1089/thy.2012.0392)

Pellegriti G, Frasca F, Regalbuto C, Squatrito S \& Vigneri R 2013 Worldwide increasing incidence of thyroid cancer: update on epidemiology and risk factors. Journal of Cancer Epidemiology 2013 965212. (doi:10.1155/2013/965212)

Rosai J, Zampi G \& Carcangiu ML 1983 Papillary carcinoma of the thyroid: A discussion of its several morphologic expressions with particular emphasis on the follicular variant. American Journal of Surgery 7 809-817. (doi:10.1097/00000478-198307080-00010)

Shi X, Liu R, Basolo F, Giannini R, Shen X, Teng D, Guan H, Shan Z, Teng W, Musholt TJ, et al. 2016 Differential clinicopathological risk and prognosis of major papillary thyroid cancer variants. Journal of Clinical Endocrinology and Metabolism 101 264-274. (doi:10.1210/ jc.2015-2917)

Siegel R, Naishadham D \& Jemal A 2013 Cancer statistics, 2013. CA: A Cancer Journal for Clinicians 63 11-30. (doi:10.3322/caac.21166)

Surveillance, Epidemiology, and End Results (SEER) Program (www.seer. cancer.gov) SEER*Stat Database: Incidence - SEER 13 Regs Research Data, November 2014 Sub (1992-2012) <Katrina/Rita Population Adjustment> - Linked To County Attributes - Total U.S., 1969-2013 Counties, National Cancer Institute, DCCPS, Surveillance Research Program, Surveillance Systems Branch, released April 2015, based on the November 2014 submission.

Tiwari RC, Clegg LX \& Zou Z 2006 Efficient interval estimation for age-adjusted cancer rates. Statistical Methods in Medical Research 15 547-569. (doi:10.1177/0962280206070621)

Xing M 2013 Molecular pathogenesis and mechanisms of thyroid cancer. Nature Reviews Cancer 13 184-199. (doi:10.1038/nrc3431)

Yu XM, Schneider DF, Leverson G, Chen H \& Sippel RS 2013 Follicular variant of papillary thyroid carcinoma is a unique clinical entity: A population-based study of 10,740 cases. Thyroid 23 1263-1268. (doi:10.1089/thy.2012.0453)

Received in final form 13 February 2016

Accepted 25 February 2016

Accepted Preprint published online 25 February 2016
(C) 2016 Society for Endocrinology Printed in Great Britain
Published by Bioscientifica Ltd 